\title{
Commitment to the B-lymphoid lineage depends on the transcription factor Pax5
}

\author{
Stephen L. Nutt ${ }^{\dagger} \dagger$, Barry Heavey $\dagger$, Antonius G. Rolink $\ddagger$ \& Meinrad Busslinger $\dagger$
}

$\dagger$ Research Institute of Molecular Pathology, Dr Bohr-Gasse 7, A-1030 Vienna, Austria

$\$$ Basel Institute for Immunology, Grenzacherstrasse 487, CH-4005 Basel, Switzerland

The Pax5 gene encoding the B-cell-specific activator protein (BSAP) is expressed within the haematopoietic system exclusively in the B-lymphoid lineage, where it is required in vivo for progression beyond the pro-B-cell stage. However, Pax5 is not essential for in vitro propagation of pro-B cells in the presence of interleukin-7 and stromal cells. Here we show that pro-B cells lacking Pax5 are also incapable of in vitro B-cell differentiation unless Pax5expression is restored by retroviral transduction. Pax ${ }^{-l-}$ pro-B cells are not restricted in their lineage fate, as stimulation with appropriate cytokines induces them to differentiate into functional macrophages, osteoclasts, dendritic cells, granulocytes and natural killer cells. As expected for a clonogenic haematopoietic progenitor with lymphomyeloid developmental potential, the Pax5 $^{-/-}$pro-B cell expresses genes of different lineage-affiliated programmes, and restoration of Pax5 activity represses this lineage-promiscuous transcription. Pax5 therefore plays an essential role in B-lineage commitment by suppressing alternative lineage choices.

All types of blood cell are generated from a pluripotent haematopoietic stem cell (HSC) through developmentally restricted progenitors which undergo lineage commitment and subsequent differentiation along a single pathway. The lymphoid lineages develop through a common lymphoid progenitor (CLP) which gives rise to natural killer, B and T cells ${ }^{1}$. Early B-cell development can be dissected into different stages according to the rearrangement status of the immunoglobulin heavy-chain $(I g H)$ locus, the expression of stage-specific cell-surface markers and growth factor requirements ${ }^{2,3}$. The earliest B-lineage precursor cells carry the $I g H$ locus still in germline configuration. $D_{H^{-}} J_{H}$ recombination is subsequently initiated in pre-BI cells ${ }^{3}$, which are also known as early pro-B cells (fraction $\mathrm{B})^{2}$. These pro-B cells can be cultured in vitro on stromal cells in the presence of interleukin-7 (IL-7) and express the B-cell surface proteins $\lambda 5, \operatorname{VpreB}, \operatorname{Ig} \alpha$ and $\operatorname{Ig} \beta$ (refs 3, 4). Completion of a functional $V_{H^{-}} D J_{H}$ rearrangement results in the expression of the pre-B-cell receptor and subsequent differentiation to small pre-B cells, which are no longer responsive to pro-B-cell growth conditions ${ }^{5}$.

The initiation of B-cell development critically depends on two transcription factors; the basic helix-loop-helix proteins encoded by the E2A gene and the early B-cell factor (EBF). In the absence of either protein, $\mathrm{B}$-cell development is aborted at the earliest stage, before $D_{H^{-}} J_{H}$ rearrangement of the $I g H$ gene ${ }^{6-8}$. Moreover, forced expression of E2A and EBF in haematopoietic precursor cells revealed that these regulators cooperatively induce the transcription of several B-lymphoid-specific genes ${ }^{9,10}$. Hence, loss- and gain-offunction experiments have implicated E2A and EBF in the control of B-lineage commitment.

A third transcriptional regulator involved in early B-lymphopoiesis is the B-cell-specific activator protein (BSAP), which is encoded by the Pax 5 gene and recognizes DNA through the highly conserved paired domain characteristic of the Pax family of transcription factors $^{11}$. Within the haematopoietic system, Pax5 is exclusively expressed in the B-lymphoid lineage from the earliest detectable precursor to the mature B-cell stage ${ }^{12,13}$. In the absence of Pax5, Bcell development is arrested in the fetal liver at a similarly early stage as in $E 2 A$ and $E B F$ mutant mice, whereas it proceeds in adult bone marrow up to the early pro-B (pre-BI) cell stage ${ }^{14,15}$. B-lymphopoiesis in the bone marrow is, however, not advanced by expression of a functionally rearranged $\mu$-heavy-chain transgen $e^{5}$, indicating that

* Present address: Wellcome/CRC Institute, Tennis Court Road, Cambridge CB2 1QR, UK. the observed developmental block does not result from the inability of $\mathrm{Pax} 5^{-/-}$pro-B cells to undergo $V_{H^{-}} D J_{H}$ rearrangements at the $\operatorname{IgH}$ locus ${ }^{15}$. Pax $5^{-/-}$pro-B cells can be cultured in vitro with similar efficiency as wild-type pro-B cells, a fact that has facilitated the genetic identification of Pax 5 target genes ${ }^{15}$. BSAP (Pax5) functions both as a transcriptional activator and as a repressor, as it positively controls CD19, $m b-1(\operatorname{Ig} \alpha), N-m y c$ and Lef-1 expression and negatively regulates $P D-1$ transcription ${ }^{16}$. Apart from these target genes, Pax5-deficient and wild-type pro-B cells do not significantly differ in the expression of $50 \mathrm{~B}$-cell-associated genes analysed ${ }^{16}$. This observation suggested that pro-B cells, even in the absence of Pax5, still undergo commitment to the B-lymphoid lineage. However, investigating the development potential of $\mathrm{Pax}^{-/-}$pro-B cells, we now show that Pax5, instead of E2A and EBF, is the critical transcription factor involved in B-lineage commitment.

\section{Pax5 $^{-I-}$ pro-B cells lack B-lymphoid potential}

The RAG2 mutation also blocks B-cell development at the pro-Bcell stage in vivo ${ }^{17}$, like the Pax 5 mutation ${ }^{15}$, but it is compatible with
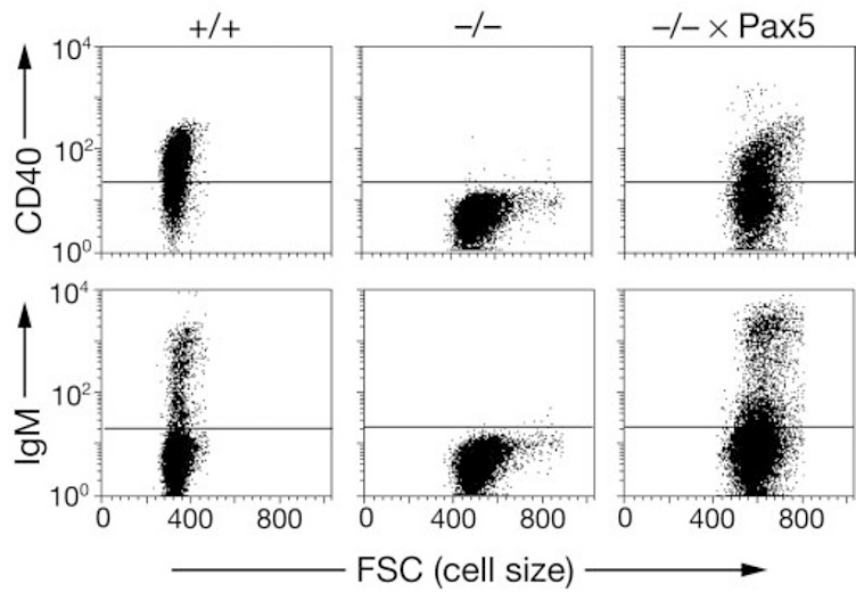

Figure 1 Pax5 is essential for in vitro differentiation of B lymphocytes. Wild-type and Pax $5^{-/-}$pro-B cells expressing a retroviral $b c / 2$ gene were cultured in the absence of stromal cells and IL-7 for three days in IMDM medium alone followed by flow cytometric analysis. Grating for $\mathrm{CD} 19^{+}$cells was used to select the Pax $5^{-/-}$pro-B cells infected with a BSAP (Pax5)-expressing retrovirus ${ }^{16}$. FSC, forward scatter. 
differentiation of these cells to mature B-lymphocytes in vitro ${ }^{18}$. We have therefore examined the in vitro differentiation potential of $\mathrm{Pax}^{-/-}$pro-B cells by culturing them for three days without IL-7 and stromal (ST2) cells. To promote cell survival upon IL-7 withdrawal $^{18}$, a retroviral bcl2 gene was introduced into Pax $5^{-1-}$ and wild-type pro-B cells. In the absence of IL-7 and ST2 cells, the wild-type cells complete the rearrangement of their immunoglobulin genes and differentiate to small CD $40^{+} \operatorname{IgM}^{+} \mathrm{B}$ cells ${ }^{18}$ (Fig. 1). In contrast, $\mathrm{Pax}^{-/-}$cells do not express any markers associated with late differentiation (Fig. 1; and data not shown). However, Pax $5^{-l-}$ pro-B cells infected with a Pax5-expressing retrovirus can differentiate into B cells in vitro (Fig. 1). Hence, the Pax5 mutation arrests B-cell development at the same early stage in vitro as in vivo, and this developmental block can be overcome by retrovirus-mediated expression of Pax5.

\section{Pax5 ${ }^{-I-}$ pro-B cells are uncommitted}

While growing pro-B-cell clones, we found that $\mathrm{Pax} 5^{-1-}$ pro-B cells, in contrast to wild-type pro-B cells, could survive for up to three weeks without medium change. Although the culture medium was depleted of IL-7 during this time, some $\mathrm{Pax}^{-1-}$ cells underwent a characteristic change in morphology and subsequently continued to proliferate. Whereas $P a \times 5^{-/-}$pro-B cells grown in IL-7 are small and refractile, and contain little cytoplasm (Fig. 2a), these cells became larger and irregular in shape upon IL-7 depletion (Fig. 2b).

To systematically investigate this phenomenon, we sorted individual pro-B cells into single wells of a 96-well plate, scored the growth of pro-B-cell colonies after 10 days in IL-7 medium and then monitored the frequency with which these colonies changed their morphology as IL-7 became limiting (Fig. 2c). For this purpose, pro-B cells were either freshly isolated from the bone marrow of $P a x 5^{-/-}, R A G 2^{-/-}$and wild-type mice (ex vivo) or were sorted from established pro-B cell cultures (in vitro). The $\mathrm{Pax}^{-/-}$pro-B-cell clones changed their morphology at a high frequency $(64-87 \%)$, irrespective of $b c l 2$ expression, whereas the wild-type and $R A G 2^{-I-}$ pro-B cells did not show this morphology change (Fig. 2c). Our interpretation of these results is that the wild-type and $R A G 2^{-1-}$ pro-B cells have undergone B-lineage commitment and can therefore only differentiate to non-proliferating B-cells upon IL-7
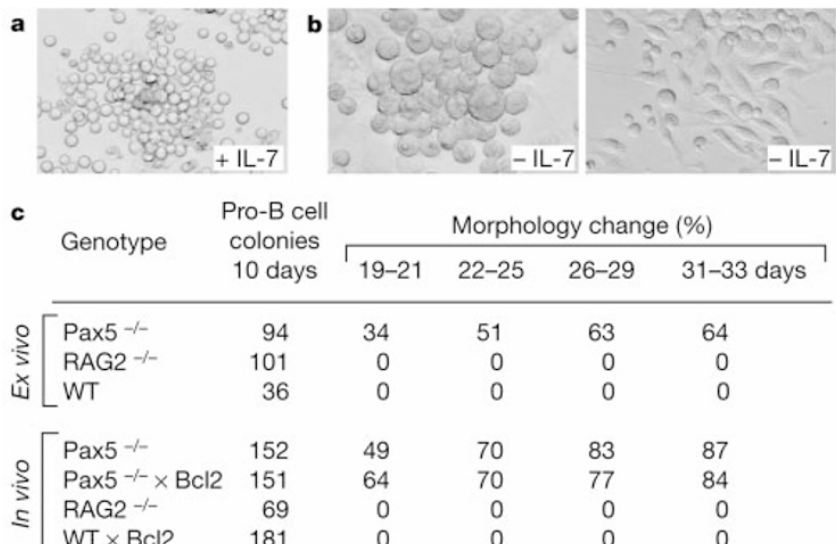

Pro-B cell 10 days

Morphology change (\%)

\begin{tabular}{|c|c|c|c|}
\hline $19-21$ & $22-25$ & $26-29$ & $31-33$ days \\
\hline 34 & 51 & 63 & 64 \\
\hline 0 & 0 & 0 & 0 \\
\hline 0 & 0 & 0 & 0 \\
\hline
\end{tabular}

$\begin{array}{rrr}49 & 70 & 83 \\ 64 & 70 & 7 \\ 0 & 0 & \\ 0 & 0 & \end{array}$

87

84

0

0

Figure 2 Pax5-deficient pro-B cells are not restricted to the B-lymphoid lineage. a, Confocal microscopic image of a $\mathrm{Pax}^{-1-}$ pro-B-cell colony after 10 days in IL-7 medium. $\mathbf{b}$, Altered morphology of two representative colonies of the same pro-B-cell culture 30 days after the last medium change. c, Statistical analysis. Individual B220 ${ }^{+} \mathrm{C}^{-}$ $\mathrm{Kit}^{+}$pro-B cells, which were isolated by single-cell sorting directly from the bone marrow (ex vivo) or from established pro-B-cell cultures (in vitro) of the indicated genotypes, were grown on stromal ST2 cells in IL-7 medium, scored for colony formation at day 10 and subsequently examined for altered morphology at 3-day intervals (without medium change). The $b c / 2$ gene was introduced by retroviral transduction. WT, wild-type.

withdrawal ${ }^{18}$ (Fig. 1). However, under the same conditions Pax $5^{-1-}$ pro-B cells cannot develop along the B-lymphoid lineage (Fig. 1). Instead, they appear to differentiate along other haematopoietic pathways, indicating that these cells are not committed to the Blymphoid lineage.
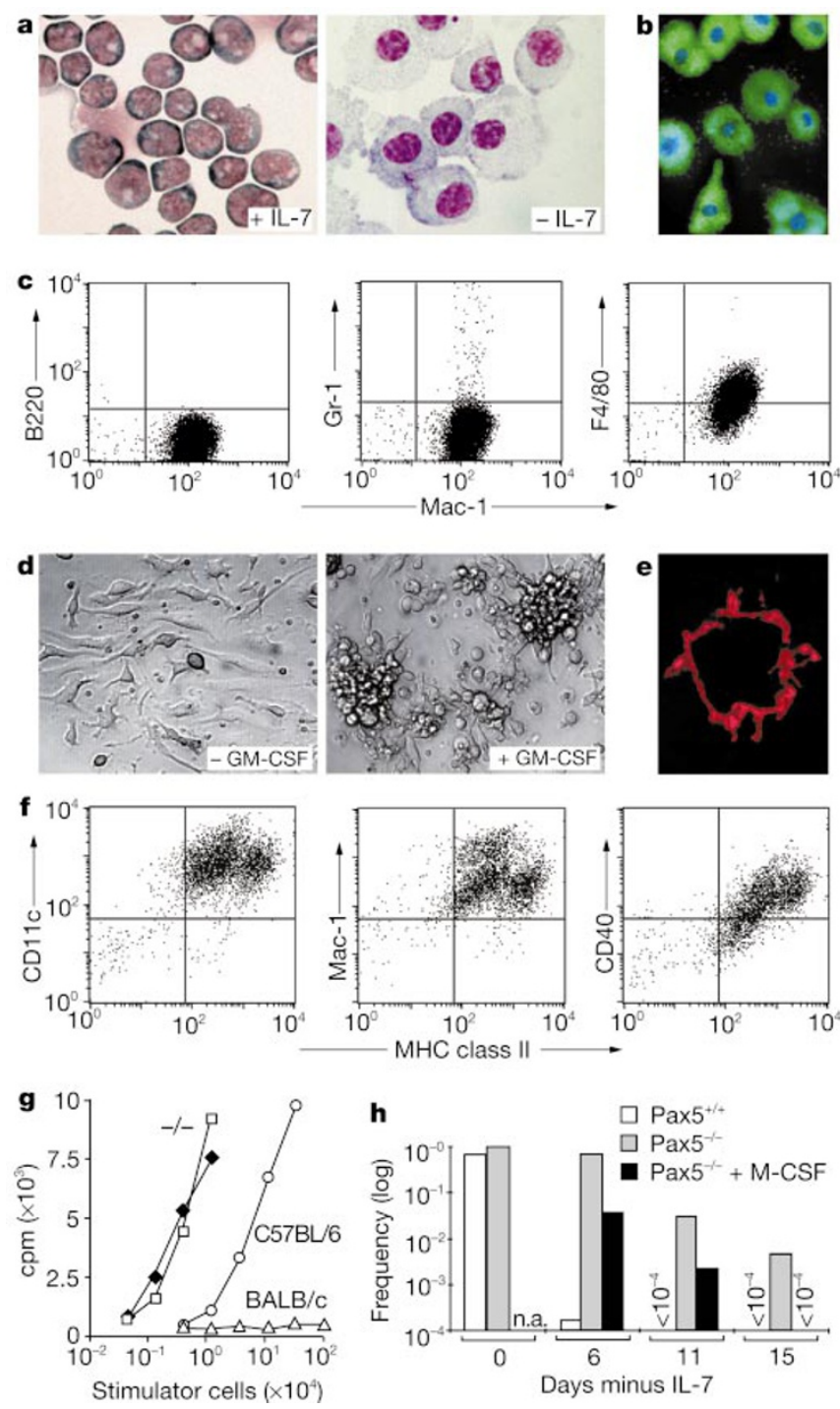

Figure 3 In vitro generation of macrophages and dendritic cells. a, May-GrünwaldGiemsa staining of cytospin preparations of $P a \times 5^{-/-}$pro-B cells cultured for 14 days on ST2 cells with or without IL-7. b. Phagocytosis. In vitro differentiated Pax $5^{-1-}$

macrophages were incubated with FITC-labelled $E$. coli. c. Flow cytometric analysis of macrophages derived from $P a x 5^{-/-}$pro-B cells. d, Confocal microscopic images of Pax $5^{-/}$pro-B cells grown for 16 days on ST2 cells with or without GM-CSF. e, MHC class II expression on cells differentiated in GM-CSF medium and stained with an anti-MHC class II antibody. f, Flow cytometric analysis of dendritic cells derived from Pax $5^{-/-}$pro-B cells. The cells shown in $\mathbf{c}$ and $\mathbf{f}$ were grated on large size. $\mathbf{g}$, Mixed leukocyte reaction. Increasing doses of $\gamma$-irradiated stimulator cells (cells per well) were incubated for four days with $2.5 \times 10^{5}$ responder cells isolated from lymph nodes of BALB/c mice. $\left[{ }^{3} \mathrm{H}\right]$ thymidine incorporation (c.p.m.) was measured during the last $14 \mathrm{~h}$ of incubation. Dendritic cells derived from two Pax $5^{-/-}$pro-B-cell lines (squares, diamonds) and splenocytes from allogeneic C57BL/6 (circles) or syngeneic BALB/c mice (triangles) were used as stimulator cells. $\mathbf{h}$, Kinetic analysis of myeloid commitment. Bcl2-expressing proB cells deprived of IL-7 and ST2 cells were cultured with or without M-CSF. Viable cells were assessed for their ability to be recloned as pro-B cells in the presence of IL-7 and ST2 cells by limiting dilution analysis ${ }^{4}$. The re-cloning frequency is indicated relative to that of $P a \times 5^{-/-}$pro-B cells at day 0. n.a., not applicable. 


\section{In vitro differentiation along monocytic lineages}

The morphology of the cells shown in Fig. 2b was suggestive of myeloid cell types, in agreement with the fact that ST2 cells produce the cytokine macrophage colony-stimulating factor $(\mathrm{M}-\mathrm{CSF})^{19}$. We therefore assessed the potential of $\mathrm{Pax}^{-/-}$pro-B cells to develop into macrophages by culturing them for 10-14 days on ST2 cells (in the absence of IL-7) followed by propagation in M-CSF (with or without ST2 cells). Cells differentiated in this manner displayed the characteristic morphology of enlarged vacuolar macrophages (Fig. 3a) and were positive for the macrophage markers Mac-1 and F4/80, but did not express the B-cell protein B220 and granulocyte marker Gr-1 (Fig. 3c). The same cells efficiently phagocytosed fluorescently labelled Escherichia coli (Fig. 3b), indicating that $\mathrm{Pax}^{-/-}$pro-B cells can differentiate into mature macrophages.

Using a cell-cloning assay, we next investigated the kinetics with which $\mathrm{Pax}^{-l-}$ pro-B cells become committed to the myeloid lineage. Bcl2-expressing wild-type and $\mathrm{Pax}^{-1-}$ pro-B cells were cultured in the absence of IL-7 and ST2 cells or in the presence of M-CSF for defined times. Subsequently, the proportion of viable cells that could be re-cloned as pro-B cells upon re-addition of stromal cells and IL-7 was determined by limiting dilution analysis. Wild-type pro-B cells rapidly lost their ability to be re-cloned (Fig. $3 \mathrm{~h}$ ) owing to efficient differentiation along the B-lymphoid lineage ${ }^{4}$. In contrast, the $\mathrm{Pax}^{-1-}$ pro-B cells, which are not yet committed to a lineage-specific programme, could be re-cloned even after 15 days of IL-7 and stromal cell withdrawal (Fig. 3h). M-CSF addition, however, reduced the cloning frequency of $\mathrm{Pax}^{-1-}$ pro-B cells 10 fold after six days and completely abolished it after 15 days (Fig. $3 \mathrm{~h}$ ). Hence, most cells initiated differentiation along the myeloid lineage and thus lost their IL-7 responsiveness within six days of M-CSF stimulation.

Dendritic cells also develop in the bone marrow from the same monocyte precursor as the macrophages, but require the cytokine granulocyte-macrophage CSF (GM-CSF) instead of M-CSF for terminal differentiation ${ }^{20,21}$. To generate dendritic cells from $\mathrm{Pax}^{-/-}$ pro-B cells, we applied a similar strategy as for macrophage development except that GM-CSF was added in the final differentiation step. After 16 days of GM-CSF stimulation, the cells were prolifer-
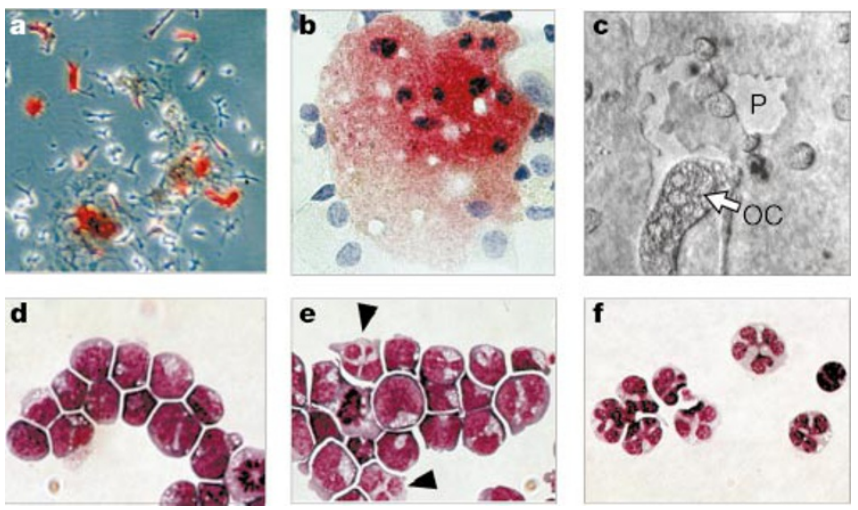

Figure 4 In vitro differentiation of osteoclasts and granulocytes. a, TRAP staining (red) of $\mathrm{Pax}^{-1-}$ cells cultured for two weeks on TRANCE-expressing ST2 cells. b. Multinucleated morphology of TRAP ${ }^{+}$cells. Differentiated Pax $5^{--}$cells were stained for TRAP activity (red) followed by counterstaining with haematoxylin (revealing the nuclei in blue) and cytospin centrifugation. c, Bone resorption. Osteoclasts derived from $\mathrm{Pax}^{-/}$pro-B cells were incubated on an osteologic bone disc with ST2-TRANCE cells. A multinucleated osteoclast $(\mathrm{OC})$ is shown with the pits $(\mathrm{P})$ resulting from its bone-resorbing activity. d-f, Cytospin preparations of May-Grünwald-Giemsa-stained cells. Pax $5^{-/}$pro-B cells initially grown in IL-7 medium (d) were cultured in the presence of IL-3, IL-6 and SCF for three weeks (e) and subsequently in G-CSF medium alone for six days (f). Granulocytes were identified by their segmented nuclear morphology (arrowhead in $\mathbf{e}$ ). ating in small aggregates characteristic of dendritic cells ${ }^{20}$, whereas only macrophages grew in the absence of GM-CSF (Fig. 3d). Mature dendritic cells are antigen-presenting cells characterized by a stellate shape with multiple cytoplasmic projections and by high levels of MHC class II protein on their surfaces ${ }^{20,21}$. The $\operatorname{Pax}^{-1-}$ cells, differentiated in response to GM-CSF, exhibited the characteristic cell morphology and MHC class II expression (Fig. 3e) as well as the expected cell-surface phenotype (MHC-II ${ }^{\text {high }} \mathrm{CD} 11 \mathrm{c}^{+} \mathrm{Mac}-$ $1^{+} \mathrm{CD} 40^{+} \mathrm{CD}^{-} \mathrm{B} 220^{-}$) of mature dendritic cells (Fig. 3f; and data not shown). These cells were further identified as dendritic cells by their ability to stimulate T-cell proliferation in a mixed leukocyte reaction (Fig. 3g). In this assay, allogeneic splenocytes of C57BL/6 mice elicit a proliferative response in $\mathrm{T}$ cells of BALB/c mice, in contrast to syngeneic splenocytes (Fig. $3 \mathrm{~g}$ ). Importantly, the Pax $5^{-1-}$ dendritic cells activated the $\mathrm{T}$ cells with a 10-30-fold higher efficiency then allogeneic splenocytes (Fig. 3g), in agreement with the fact that dendritic cells are significantly more potent than splenocytes in stimulating T-cell proliferation ${ }^{21}$. $\mathrm{Pax}^{-1-}$ pro-B cells can therefore also differentiate to become functional dendritic cells.

Osteoclasts constitute a third lineage which originates from a common monocyte precursor. Differentiation to bone-resorbing osteoclasts is controlled by the cytokine TRANCE (also known as OPGL, ODF or RANKL), which is presented on activated osteoblasts $^{22}$. Following IL-7 withdrawal, we therefore cultured Pax $5^{-/-}$pro-B cells on stromal ST2 cells which ectopically expressed TRANCE. Under these conditions, the pro-B cells differentiated within two weeks into cells expressing the osteoclast-specific enzyme tartrate-resistant acid phosphatase (TRAP; Fig. 4a). These differentiated cells had the multinucleated morphology of mature osteoclasts (Fig. 4b) and formed resorption lacunae on artificial bone substrates (Fig. 4c). Hence, $\mathrm{Pax}^{-1-}$ pro-B cells can also develop into functional osteoclasts.

\section{Differentiation into granulocytes}

Myeloid progenitors also give rise to cell types of the neutrophil lineage which characteristically contain a highly segmented nucleus and differentiate under the influence of the cytokines IL-6 and granulocyte CSF (G-CSF) ${ }^{23}$. To test the granulocytic potential of $\mathrm{Pax}^{-1-}$ pro-B cells, we cultured these cells for three weeks with the multilineage cytokines IL-3, IL-6 and stem-cell factor (SCF). Under these conditions, a small percentage of the $P a \times 55^{-1-}$ cells differentiated into granulocytic cells, as shown by their segmented nuclear morphology (Fig. 4e). Homogeneous differentiation to granulocytes was achieved by replacing the multilineage cytokines with GCSF for six days (Fig. 4f). Pax $5^{-1-}$ pro-B cells can, therefore, also differentiate along the granulocytic lineage.
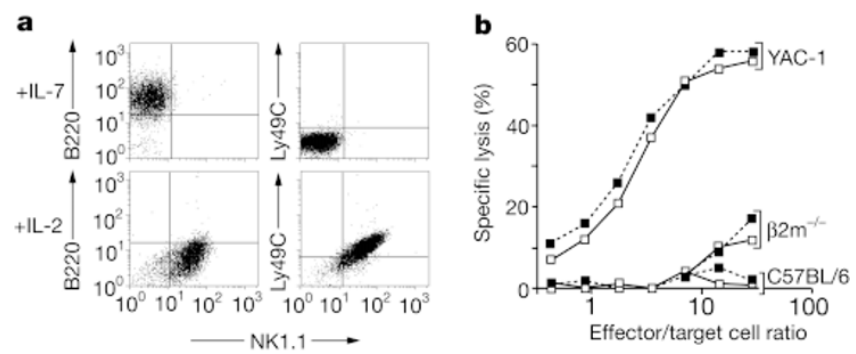

Figure 5 Generation of mature NK cells. a, Flow cytometric analysis of Pax $5^{-1-}$ pro-B cells cultured for nine days with IL-2 and ST2 cells. b. Cytoxicity. Cells of two Pax $5^{-/-}$ pro-B-cell lines (closed and open symbols) were differentiated in IL-2 for nine days and assayed for their cytotoxicity against YAC-1 cells and LPS-treated splenocytes from wild-type C57BL/6 or $\beta_{2} \mathrm{~m}^{-1-}$ mice $\mathrm{e}^{27}$. Cytolytic assays were performed at the indicated effector to target cell ratios. 


\section{Differentiation into natural killer cells}

The myeloid differentiation experiments unequivocally show that $P a \times 5^{-1-}$ pro-B cells are not committed to the B-lymphoid lineage. Hence, the question arose of whether these cells also have properties of the recently described CLP and could thus give rise to natural killer (NK) cells ${ }^{1}$. NK-cell development critically depends on IL-15 (ref. 24), which can, at least in vitro, be substituted by IL-2 (ref. 25). To assess their NK-cell potential, the $P a x 5^{-1-}$ pro-B cells were therefore cultured with IL-2 and stromal cells. Within 10 days, the $\mathrm{Pax}^{-/-}$cells switched off expression of the B-cell protein B220, initiated synthesis of the NK-cell markers NK1.1, Ly49C and 2B4 and were identified as $\mathrm{NK}$ cells by their cell-surface phenotype $\left(\mathrm{NK} 1.1^{+} \mathrm{Ly}_{49 \mathrm{C}^{+}} 2 \mathrm{~B} 4^{+}\right.$IL-2R $\alpha^{\text {low }}$ IL-2R $\beta^{\text {low }} \mathrm{Mac} 1^{\text {low }} \mathrm{CD} 3 \epsilon^{-} \mathrm{CD} 4^{-}$ $\mathrm{CD}^{-}$B220- ; Fig. 5a and data not shown).

NK cells contribute to innate immunity by killing host cells that fail to express MHC class I proteins on their surfaces as a consequence of viral infection or tumorigenesis ${ }^{26}$. We therefore measured the cytolytic activity of in vitro differentiated $\mathrm{Pax}^{-/-}$cells in a ${ }^{51} \mathrm{Cr}$ release assay, using the sensitive YAC-1 tumour cells or LPS-stimulated lymphoblasts from $\beta_{2}$-microglobulin-deficient $\left(\beta_{2} m^{-l-}\right)$ mice, which lack MHC class I cell-surface expression ${ }^{27}$. Both target cells were lysed in a dose-dependent manner by NK cells derived from $\mathrm{Pax}^{-/-}$pro-B cells, whereas LPS-activated target cells from a syngeneic C57BL/6 mouse were resistant to lysis (Fig. 5b). Hence, $P a \times 5^{-1-}$ pro-B cells can differentiate into functional NK cells.

Under all in vitro differentiation conditions analysed, we never detected $\mathrm{Pax}^{-/-}$cells that expressed surface antigens characteristic of the T-lymphoid lineage, in agreement with the fact that the complex inductive microenvironment of the thymus is required for early T-cell development. When injected into $R A G 2^{-/-}$mice, Pax5deficient pro-B cells were, however, able to fully reconstitute T-cell development in vivo ${ }^{28}$. Therefore, $\mathrm{Pax}^{-1-}$ pro-B cells can also give rise to lymphocytes except for $\mathrm{B}$ cells.

\section{In vivo myeloid development of Pax $^{-I-}$ pro-B cells}

The observation that injected $\mathrm{Pax}^{-/-}$pro-B cells home to the bone
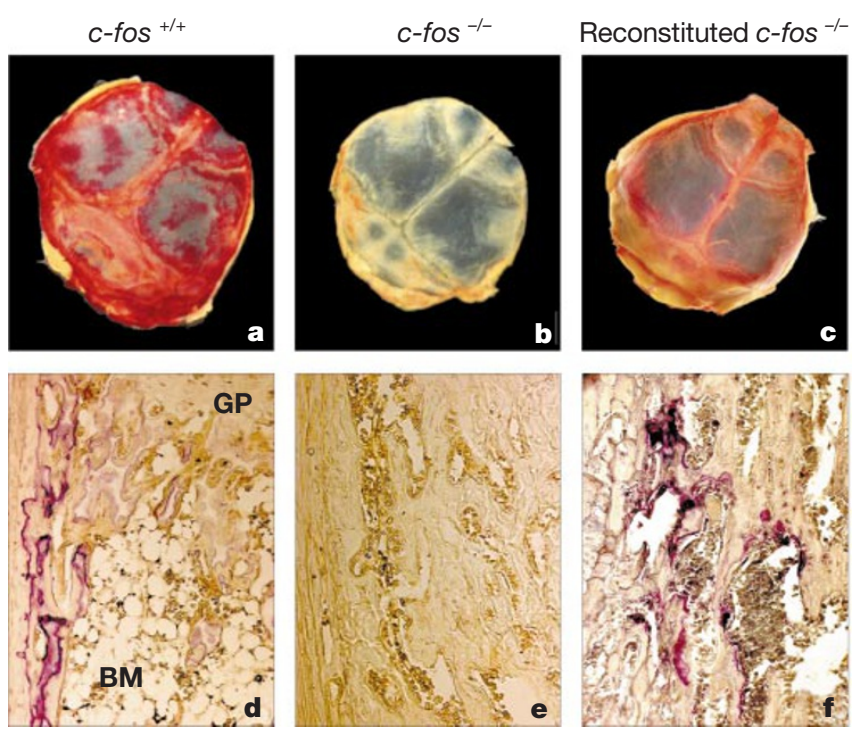

Figure 6 Development of osteoclasts from $P a x 5^{-/-}$pro-B cells in vivo. Identification of osteoclasts in the calvariae $(\mathbf{a}-\mathbf{c})$ and long bones $(\mathbf{d}-\mathbf{f})$ of 4 -week-old mice. TRAP staining (red) was used to detect osteoclasts on the calvarial surface and in sections of the tibia of wild-type $(\mathbf{a}, \mathbf{b}), \mathrm{c}^{-} \mathrm{fos}^{-/-}(\mathbf{b}, \mathbf{e})$ and reconstituted c- $\mathrm{fos}^{-1-}(\mathbf{c}, \mathbf{f})$ mice. For reconstitution, $10^{7} \mathrm{Pax}^{-1-}$ pro-B cells and $10^{7}$ splenocytes, isolated from an adult $\mathrm{c}-\mathrm{fos}^{-1-}$ mouse, were co-injected intraperitoneally into lethally irradiated $\mathrm{c}-\mathrm{fos}^{-1-}$ mice one day after birth. Four weeks later, osteoclast formation was analysed by TRAP histochemistry. BM, bone marrow cavity; GP, growth plate. marrow and undergo self-renewal in $R A G 2^{-1-}$ mice ${ }^{28}$ raises the question of whether these cells can also differentiate in vivo into myeloid cells, in addition to T-lymphocytes. However, we could not detect the formation of myeloid cell types in reconstituted $R A G 2^{-/-}$ mice ${ }^{28}$, suggesting that the $P a \times 5^{-1-}$ pro-B cells cannot efficiently compete with endogenous myeloid progenitors. For this reason, we analysed the in vivo myeloid potential of $\mathrm{Pax}^{-1-}$ pro-B cells in the $\mathrm{c}-\mathrm{fos}^{-1-}$ mouse, which has a developmental defect in the osteoclast lineage $\mathrm{e}^{29,30}$. Consequently, $\mathrm{c}-\mathrm{fos}^{-1-}$ mice develop osteopetrotic bones and, owing to the absence of a bone marrow cavity, exhibit extramedullary haematopoiesis in the spleen ${ }^{30}$. As transplantation of $\mathrm{Pax}^{-1-}$ pro-B cells alone cannot rescue lethally irradiated mice (data not shown), we performed competitive reconstitution experiments by injecting Pax $5^{-/-}$pro-B cells together with $\mathrm{c}-\mathrm{fos}^{-1-}$ splenocytes into lethally irradiated, newborn $\mathrm{c}-\mathrm{fos}^{-1-}$ mice. Four weeks after cell transfer, osteoclasts derived from $\mathrm{Pax} 5^{-/-}$pro-B cells could be detected by TRAP staining on the surface of the calvariae of three reconstituted c- fos $^{-1-}$ mice (Fig. 6c), as in wild-type mice (Fig. 6a), whereas calvarial osteoclasts were never found in untreated $\mathrm{c}-\mathrm{fos}^{-1-}$ mice (Fig. 6b). However, the reconstituted ${\mathrm{c}-f o s^{-1-}}^{-}$mice showed neither tooth eruption nor development of a bone marrow cavity in the long bones (Fig. 6f). A few clusters of TRAP ${ }^{+}$osteoclasts were nevertheless observed in the osteopetrotic bones of the reconstituted c- $\mathrm{fos}^{-1-}$ mice (Fig. 6f), in contrast to untreated c-fos ${ }^{-1-}$ mice (Fig. 6e). Hence, the injected $\mathrm{Pax}^{-/-}$pro-B cells gave rise to only a partial rescue of the c-fos mutant phenotype, possible owing to still inefficient competition with myeloid progenitors derived from the ${\mathrm{c}-f o \mathrm{~S}^{-1-}}$ HSC. Nevertheless, these experiments show that Pax5 $5^{-1-}$ pro-B cells can differentiate into at least one myeloid cell type in vivo.

\section{The multilineage potential is clonal}

During this study, we tested the myeloid and lymphoid differentiation potential of five independently derived $\mathrm{Pax}^{-/-}$pro-B-cell pools, of which three were infected with a $b c l 2$-expressing retrovirus. All five pro-B-cell lines developed into macrophages in vitro and $\mathrm{T}$ cells a

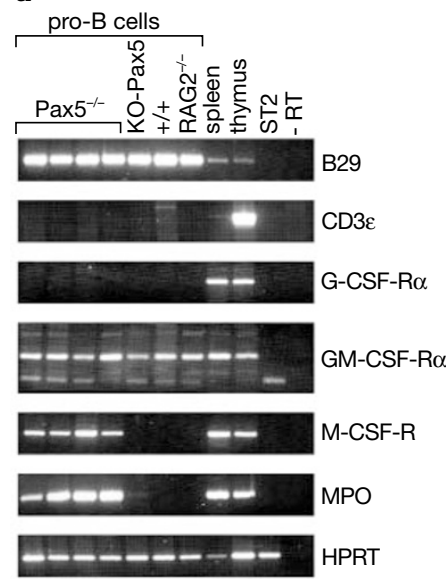

b
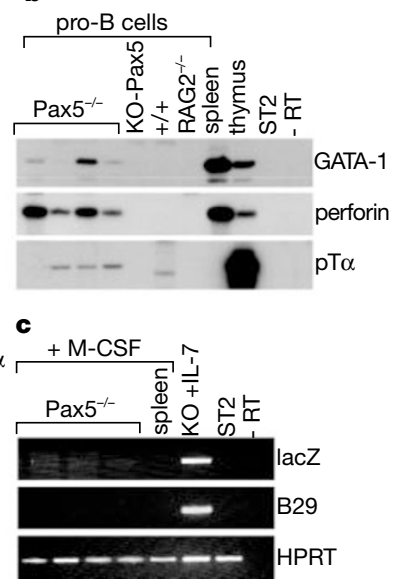

Figure 7 Lineage-promiscuous gene expression in Pax $5^{-/-}$pro-B cells. a, b, RT-PCR analysis. The following pro-B cells grown on stromal ST2 cells in the presence of IL-7 were analysed: four independent $P a \times 5^{--}$pro-B cell lines, $P a \times 5^{-1-}$ pro-B cells reconstituted with a Pax5-expressing retrovirus (KO-Pax5) and pro-B cells established from wild-type $(+/+)$ and $R A G 2^{-/-}$bone marrow. The PCR products were visualized on agarose gels by ethidium bromide staining (a) or Southern blot analysis $(\mathbf{b})$. The quantity of input cDNA was normalized by analysing the control HPRT transcript. No reverse transcriptase was added in lane - RT. c, Loss of Pax5 expression upon myeloid differentiation. Pax $5^{-/-}$proB cells and wild-type splenocytes were cultured for 10 days with M-CSF and ST2 cells. Pax5 expression was subsequently monitored by RT-PCR with primers from Pax5 exon $1 \mathrm{~A}$ and lacZ sequences inserted in the targeted Pax5 locus $^{14}$. Pax $5^{-1-}$ pro-B cells grown in $\mathrm{IL}-7$ medium (KO + IL-7) were used as positive control. 
in vivo, indicating that the ability to differentiate along the myeloid and lymphoid lineages is an intrinsic property of all $\mathrm{Pax}^{-1-}$ pro-B cells, irrespective of $b c l 2$ expression. However, it could be argued that, despite their growth in IL-7, the $\mathrm{Pax} 5^{-/-}$pro-B-cell pools may correspond to a heterogeneous mixture of lineage-committed progenitors. To exclude this possibility, we have analysed the differentiation potential of eight subclones which were generated by single-cell sorting of $\mathrm{Pax} 5^{-\gamma-}$ pro-B-cell pools. In the appropriate environment, all eight subclones efficiently developed into macrophages, osteoclasts, dendritic cells (in vitro) and T cells (in vivo). The differentiation into granulocytes and NK cells was variable, possibly indicating either that our in vitro differentiation conditions were not optimal for these two lineages or that in vitro cultured $\mathrm{Pax}^{-1-}$ pro-B cells gradually lose the capacity to generate these two cell types. Nevertheless, our results unequivocally show that $\mathrm{Pax}^{-/-}$ pro-B cells are multipotent haematopoietic progenitors which maintain their differentiation potential after single-cell cloning.

\section{Pax5 represses lineage-promiscuous transcription}

Multipotent haematopoietic progenitors simultaneously express genes from different lineage-affiliated programs, in a process known as 'multilineage priming' ${ }^{31}$. Consistent with its progenitor status, the $\mathrm{Pax}^{-1-}$ pro-B cell is therefore expected to exhibit lineagepromiscuous gene expression, even though $\mathrm{Pax} 5^{-/-}$pro-B cells differ only minimally from committed wild-type pro-B cells in the expression of $50 \mathrm{~B}$-cell-associated genes analysed ${ }^{16}$. To investigate this issue, we used the polymerase chain reaction with reverse transcription (RT-PCR) to compare the expression of different lineage-specific genes between uncommitted $\mathrm{Pax}^{-/-}$pro-B-cell lines and committed pro-B cells derived either by retrovirusmediated reconstitution of Pax5 expression or by isolation from wild-type and $R A G 2^{-1-}$ bone marrow (Fig. 7a, b). According to their expression pattern, the different genes could be grouped into three categories (Fig. 7; and data not shown). The transcripts of several genes, including CD3€ (T-lymphoid), G-CSF-R $\alpha$ (granulocytic) and $\beta^{\text {maj }}$-globin (erythroid), were not detected in any pro-B-cell line, whereas they could be readily amplified from control tissues. Other genes were equally expressed in pro-B cells of all genotypes. This category comprises the genes B29 (B-lymphoid), GM-CSF-R $\alpha$ (myeloid), EPO-R (erythroid), Tcf-1 (T-lymphoid) and GATA-3 (Tlymphoid), although the last two transcription factor genes were expressed only at a very low level. Some genes, on the other hand, were exclusively expressed in $\mathrm{Pax} 5^{-/-}$pro-B cells, but not in any of the committed pro-B cells. This group includes the relatively abundant transcripts of the myeloid M-CSF receptor (M-CSF-R; c-fms) and myeloperoxidase (MPO) genes as well as the rare messenger RNAs of the GATA-1 (erythroid), perforin (NK cell) and $p T \alpha$ (T lymphoid) genes. Importantly, the lineage-promiscuous expression of these genes was efficiently repressed in $\mathrm{Pax}^{-/-}$ pro-B cells by restoring Pax 5 activity using retroviral transduction (Fig. 7a, b).

We next investigated whether the transcription of B-cell-specific genes such as $B 29(\operatorname{Ig} \beta)$ and Pax5 is repressed in $P a x 5^{-1-}$ pro-B cells upon entry into the myeloid lineage. Ten days after replacement of IL-7 with M-CSF, four independent $\mathrm{Pax} 5^{-/-}$cell lines efficiently downregulated the expression of $B 29$ as well as of the lac $Z$ gene inserted in the targeted Pax5 locus (Fig. 7c). In summary, our data indicate that an essential fucntion of Pax 5 in B-lineage commitment is to repress the lineage-promiscuous transcription of non- $\mathrm{B}$ lymphoid genes. Conversely, transcription of the Pax5 gene is itself inactivated upon differentiation to other haematopoietic lineages.

\section{Discussion}

Differentiation of the HSC into distinct blood cell types is thought to progress through intermediate progenitor cells with restricted developmental potential. This view of haematopoiesis is challenged by our finding that the Pax $5^{-/-}$pro-B cell possesses, at least under in vitro conditions, a broad developmental potential similar to that of the HSC itself. So far, however, we have been unable to differentiate the $P a x 5^{-/-}$pro-B cell along the erythroid and megakaryocytic lineages, which may reflect a more restricted developmental capacity of this cell or our failure to define appropriate differentiation conditions for these lineages. Moreover, $\mathrm{Pax} 5^{-/-}$pro-B cells have so far failed to reconstitute the entire haematopoietic system in transplantation experiments ${ }^{28}$. In the absence of this characteristic property of the HSC, the Pax $5^{-/-}$pro-B cell must be classified as a haematopoietic progenitor cell with broad lymphoid and myeloid differentiation potential.

A close developmental connection between the lymphoid and myeloid lineages has been demonstrated by the identification of a Bcell/macrophage-restricted precursor, on the basis of several criteria $^{32}$. First, certain human acute leukaemias co-express B-cell and macrophage characteristics, indicating that they may be derived from a transformed progenitor cell of both lineages ${ }^{33}$. Second, mice expressing the $\mathrm{E} \mu-m y c$ transgene generate immature lymphomas that can differentiate along the B-lymphoid and macrophage lineages ${ }^{34,35}$. Third, several immortalized pre-B-cell lines can be converted into macrophages ${ }^{36-38}$. Finally, the murine fetal liver contains a small subpopulation of clonogenic B-cell/macrophage progenitors $^{39}$. Fetal liver cells with an expanded lymphomyeloid potential have been identified by several groups by varying the conditions of their in vitro differentiation assays ${ }^{40-42}$. A progenitor cell with a similar developmental capacity has so far not been isolated from mouse bone marrow ${ }^{1}$, whereas human bone marrow contains cells with the potential to differentiate along the $\mathrm{T}, \mathrm{B}, \mathrm{NK}$ and dendritic cell lineages ${ }^{43}$. The interpretation of these studies is, however, complicated by the fact that the isolated progenitor cells are rare, correspond to only a transition stage in haematopoietic development and cannot be expanded long-term in vitro in an uncommitted state, thus precluding any detailed analysis. In contrast, the $P a x 5^{-1-}$ pro-B cell can be propagated in vitro as an uncommitted haematopoietic progenitor. Moreover, this cell originates from the bone marrow and, except for B-cell development, exhibits the full spectrum of myeloid and lymphoid differentiation.

The development of lymphocytes, but not of myeloid cells, is critically dependent on IL-7, which promotes the proliferation and/ or survival of lymphoid precursors ${ }^{44}$. Expression of the IL-7 receptor was used as a criterion for isolating the CLP from mouse bone marrow $^{1}$. This CLP has the capacity to rapidly reconstitute $\mathrm{B}, \mathrm{T}$ and NK cells in vivo, but lacks myeloid differentiation potential in vivo and in vitro ${ }^{1}$. A close relationship between the CLP and $\mathrm{Pax} 5^{-/-}$pro$\mathrm{B}$ cell is suggested by the fact that the $P a x 5^{-/-}$pro-B cell also expresses the IL-7 receptor $^{15}$, depends on IL-7 for in vitro propagation $^{15}$ and possesses lymphoid developmental potential. However, in contrast to the CLP, the $\mathrm{Pax} 5^{-/-}$pro-B cell can differentiate along myeloid lineages in vitro and generate osteoclasts in vivo after injection into $\mathrm{c}-\mathrm{fos}^{-\gamma-}$ mice. Given the transitory nature of the CLP, it is conceivable that, shortly after its isolation, the CLP may express Pax5 or an equivalent commitment factor of the NKand T-cell lineages, which subsequently prevents differentiation along myeloid lineages. In this view, the full developmental capacity of the CLP is only revealed under conditions that interfere with commitment to the lymphoid lineages, such as IL-7-dependent proliferation in the absence of Pax 5 function.

Multilineage priming of gene expression is a characteristic property of early haematopoietic progenitor cells ${ }^{45}$. This process ensures that genes of different haematopoietic lineages are coexpressed within a single cell, albeit often at a low level ${ }^{31}$. The lineage-promiscuous gene expression observed in the $\mathrm{Pax} 5^{-1-}$ pro-B cell thus provides molecular evidence for the progenitor status of this cell. The expression of $M-C S F-R, G M-C S F-R \alpha$ and Epo-R may also explain why the $P a x 5^{-1-}$ pro-B cell can respond to different 
cytokines. For instance, expression of the M-CSF receptor renders the $\mathrm{Pax} 5^{-/-}$pro-B cell responsive to M-CSF, which is produced by the ST2 feeder cells used for pro-B-cell culture ${ }^{19}$. IL-7 is, however, dominant over M-CSF in lymphoid precursor cells ${ }^{38}$. As a consequence, M-CSF can support monocytic differentiation and proliferation only at limiting IL-7 concentrations, which explains the observed morphology change of $\mathrm{Pax}^{-1-}$ pro-B cells in IL-7depleted medium.

Early events in lineage specification involve the control of proliferation, survival and commitment of lineage-restricted precursor cells. The loss of an entire lineage by gene targeting can therefore result from interference with any one of these processes and does not necessarily imply a role of the mutated gene in lineage commitment. A hallmark of commitment is the stabilization of a lineage-specific gene-expression programme which permanently inhibits alternative lineage choices ${ }^{45}$. Consequently, restriction of developmental plasticity provides a better criterion for defining lineage commitment. Loss-of-function experiments can therefore implicate a transcription factor in lineage commitment only if the lack of 'forward' differentiation is accompanied by maintenance of developmental plasticity in the transcription-factor-deficient progenitor cell. However, it must be possible to grow a mutant progenitor in vitro before its developmental potential can be analysed. So far, Pax5 is the only lineage-specific transcription factor to fulfil this criterion, as $\mathrm{Pax} 5^{-1-}$ pro-B cells can differentiate along multiple myeloid and lymphoid lineages with the exception of the B-cell pathway. Restoration of Pax 5 activity overcomes the B-cell developmental block, thus identifying Pax 5 as the critical B-lineage commitment factor. Surprisingly, considerable progression down the B-cell pathway is possible in the absence of commitment, as $\mathrm{Pax}^{-/-}$pro- $\mathrm{B}$ cells express many genes previously considered to be indicative of B-lineage commitment ${ }^{15,16}$. These genes include $\operatorname{Ig} \alpha(m b-1), \operatorname{Ig} \beta(B 29), \operatorname{VpreB}$ and $\lambda 5$ as well as the sterile transcripts of the $\operatorname{IgH}$ locus, all of which are under single or combinatorial control by the transcription factors E2A and $\mathrm{EBF}^{7-9,46}$. Consistently, both E2A and EBF, which act upstream of Pax5 in the genetic hierarchy of B-cell development ${ }^{7,8}$, are equally expressed in $\mathrm{Pax}^{-1-}$ and wild-type pro-B cells ${ }^{15}$. Hence, the Pax5 mutation dissociates the initial activation of $\mathrm{B}$-lineage-specific gene expression by E2A and EBF from the Pax5-dependent control of Blineage commitment. Pax5 fulfils a dual role in B-lineage commitment, as it activates the expression of B-cell-specific genes and simultaneously represses the lineage-promiscuous transcription of other haematopoietic genes. The transcriptional activation is best exemplified by CD19, whose expression is strictly dependent on Pax5 (ref. 16) and should thus be regarded as a decisive marker of B-lineage commitment. Moreover, the Pax5-dependent repression of the $M-C S F-R$ gene illustrates at the molecular level how the developmental potential is restricted at commitment by rendering $\mathrm{B}$-cell precursors unresponsive to lineage-inappropriate cytokines such as M-CSF.

A long-standing debate concerns whether lineage commitment in the haematopoietic system occurs in a cell-autonomous, stochastic manner or is controlled by instructive signals from the local environment ${ }^{47,48}$. Much attention has been paid to the role of haematopoietic growth factors in this process, but no evidence for any defect in lineage commitment has been observed despite the analysis of many growth factor gene knock-outs ${ }^{48}$. Instead, growth factors seem to perform a selective role by promoting the survival and/or proliferation of independently committed cells. We have shown that the expression of Pax5 is randomly initiated from only one of its two alleles at the onset of B-lymphopoiesis ${ }^{49}$. The transcription of a single allele indicates that the lineage commitment gene Pax5 is activated in a relatively inefficient, stochastic manner in the multipotent progenitor ${ }^{49}$. However, once this stochastic event has occurred, it restricts the various developmental options of the progenitor to the B-cell pathway. Hence, our data support the notion that B-lineage commitment is a stochastic rather than a deterministic process.

\section{Methods}

Mice

$\mathrm{Pax}^{-/-}, \mathrm{RAG2}^{-/-}$nd c- fos $^{-/-}$mice ${ }^{14,17,29}$ were maintained on the C57BL/6x129/Sv background and genotyped as described ${ }^{5,15,29}$.

\section{Pro-B cell culture}

Iscove's modified Dulbecco's medium supplemented with $50 \mu \mathrm{M} \beta$-mercaptoethanol, $1 \mathrm{mM}$ glutamine, $2 \%$ heat-inactivated fetal calf serum and $0.03 \%(\mathrm{w} / \mathrm{v})$ primatone was used for all cell-culture experiments (referred to as IMDM medium). Pro-B cells isolated from bone marrow of 2-week-old mice were cultured on $\gamma$-irradiated ST2 cells in IMDM medium containing IL-7 (ref. 15).

\section{Retroviral infection}

The retrovirus pBabe-BSAP has been described ${ }^{16}$; $\mathrm{pBabe-TRANCE}$ was provided by K. Matsuo and E. F. Wagner. Murine bcl2 cDNA was inserted into the histidinol resistance vector pMV-10 to generate a $b c l 2$-expressing retrovirus. Transfected GP + E-86 packaging cells were selected with $2.5 \mu \mathrm{g} \mathrm{ml}^{-1}$ puromycin or $10 \mathrm{mM}$ histidinol (Sigma). Pro-B cells were infected by coculture with puromycin-histidinol-resistant ST2 cells and virusproducing packaging cells followed by puromycin $\left(2.5 \mu \mathrm{g} \mathrm{ml}^{-1}\right)$ or histidinol $(1 \mathrm{mM})$ selection.

\section{Antibodies}

Anti-CD40 (FGK45.5), anti- $\mu \mathrm{H}$ (M41), anti-CD19 (1D3), anti-c-Kit (ACK4) and antiMHC class II (M5-114) antibodies were purified and biotinylated as described ${ }^{15}$. Fluorescein isothiocyanate (FITC)- and phycoerythrin (PE)-labelled anti-B220 (RA36B2), FITC-labelled anti-Ly49C (5E6) and anti-Mac-1 (M1/70), PE-conjugated CD11c (HL3) and Gr-1 (RB6-8C5), biotinylated anti-CD23 (B3B4) and anti-NK1.1 (PK136) antibodies and allophycocyanin (APC)-conjugated streptavidin were obtained from PharMingen; PE-conjugated anti-F4/80 (C1:A3-1) antibody from Serotec; and PElabelled streptavidin from Southern Biotechnology Associates.

\section{Flow cytometry}

Cells were stained and analysed on a FACSCalibur (Becton Dickinson) as described ${ }^{14}$ Biotinylated antibodies were revealed with PE- or APC-streptavidin. Myeloid cells were incubated with $10 \%$ heat-inactivated rat serum before antibody staining.

\section{Growth factors}

Murine M-CSF (used at a final concentration of $25 \mathrm{ng} \mathrm{ml}^{-1}$ ), G-CSF $\left(25 \mathrm{ng} \mathrm{ml}^{-1}\right.$ ) and SCF $\left(100 \mathrm{ng} \mathrm{ml}^{-1}\right.$ ) were purchased from R\&D Systems. All other cytokines were produced by X63 or J558L cell transfectants ${ }^{50}$ and used at a concentration of $1 \%$ (IL-7), 2\% (IL-3, IL-6), $5 \%$ (IL-2, IL-4) and 10\% (GM-CSF) conditioned supernatant.

\section{Cell morphology assay}

Individual $\mathrm{B} 220^{+} \mathrm{c}-\mathrm{Kit}^{+}$pro-B cells from bone marrow (ex vivo) or long-term pro-B-cell cultures (in vitro) were sorted by a FACSVantage TSO flow cytometer (Becton Dickinson) into single wells of 96-well plates containing ST2 cells in IL-7 medium. Colonies of small, round, refractile pro-B cells were identified after 10 days and thereafter scored for morphology changes defined by an increase in cell size, elongation and acquisition of granular vacuolated cytoplasm.

\section{Cell-cloning assay}

The cloning frequency of pro-B cells cultured on ST2 cells plus IL-7, in medium alone or in the presence of M-CSF, was assessed by limiting dilution analysis as described ${ }^{4}$. Cell numbers were normalized for viable cells (determined by trypan blue exclusion) before dilution into 96 -well plates.

\section{In vitro differentiation}

All differentiation assays were performed with in vitro cultured pro-B cells. B lymphocytes: pro-B cells were incubated at $1-3 \times 10^{6}$ cells per $\mathrm{ml}$ for three days in IMDM medium alone as described ${ }^{18}$. NK cells: pro-B cells were differentiated for 10 days in the presence of IL-2 and ST2 cells. Cytoxicity assays were performed with YAC-1 cells or LPS-stimulated splenocytes as described ${ }^{25}$. Macrophages: Pax $^{-/-}$pro-B cells were differentiated in IMDM medium with ST2 cells for 10-14 days followed by terminal differentiation in M-CSF medium for seven days. Cells were transferred to chamber slides (Nalge Nunc), incubated with $10^{7}$ FITC-labelled heat-inactivated E. coli (Molecular Probes) for $1-2 \mathrm{~h}$ at $37^{\circ} \mathrm{C}$, washed twice in PBS, fixed in $2 \%$ paraformaldehyde for $10 \mathrm{~min}$, air-dried, mounted in VectaShield (Vector Laboratories) and DABCO $\left(10 \mu \mathrm{g} \mu \mathrm{l}^{-1}\right.$, Sigma)-DAPI $\left(0.15 \mu \mathrm{g} \mu \mathrm{l}^{-1}\right.$, Sigma) solutions (1:1) and analysed on a fluorescence microscope (Zeiss Axiophot) with a CCD camera (Photometrics). Dendritic cells: $\mathrm{Pax}^{-1-}$ pro-B cells were differentiated for 16 days on ST2 cells in GM-CSF medium. For immunocytochemical analysis, cells were flushed off the stromal cell layer and stained with PE-anti-MHC class II antibodies before cytospin centrifugation and fluorescence microscopy. Differentiated cells were tested for antigen presentation by the mixed leukocyte reaction as described ${ }^{20}$. Osteoclasts: $\operatorname{Pax}^{-1}$ 
pro-B cells were cultured for two weeks with M-CSF and ST2 cells expressing TRANCE. Cells were fixed in $3.7 \%$ formaldehyde for 30 min, dried and subjected to TRAP staining (Sigma). At day 10 of differentiation, cells were transferred onto osteologic bone discs (Millenium Biologic) together with ST2-TRANCE cells in M-CSF medium containing $100 \mathrm{nM}$ dexamethasone, $10 \mathrm{mM}$ vitamin $\mathrm{D}_{3}$ and $50 \mu \mathrm{g} \mathrm{ml}^{-1}$ vitamin $\mathrm{C}$. Bone resorption was analysed after an additional 10-day incubation. Granulocytes: $\mathrm{Pax}^{-1-}$ pro-B cells were cultured for three weeks in IMDM medium containing IL-3, IL-6 and SCF and then incubated for six days in IMDM medium containing G-CSF alone. The cell morphology was analysed by May-Grünwald-Giesma staining.

\section{In vivo osteoclast development}

Newborn mice were lethally irradiated ( $800 \mathrm{rad})$ and then intraperitoneally injected with a mixture of $10^{7}$ in vitro cultured $\mathrm{Pax}^{-/-}$pro-B cells and $10^{7}$ splenocytes from an adult $\mathrm{c}-\mathrm{fos}^{-1-}$ mouse. Four weeks later, osteoclast formation was analysed by TRAP staining of isolated calvariae and histological sections of long bones ${ }^{30}$.

\section{RT-PCR}

Total RNA $(2 \mu \mathrm{g})$ from pro-B cells and lymphoid tissues was reverse-transcribed and PCRamplified as described ${ }^{49}$. The following primers were used: Pax5-lac Z, 5'-CATGGCGAGAAGCTCTTTAGTTCC-3' and 5'-TGCAAGGCGATTAAGTTGGGTAAC-3', CD $\epsilon, 5^{\prime}$ GTCTCCATCTCAGGAACCAGT- $3^{\prime}$ and $5^{\prime}$-ATAGTCTGGGTTGGGAACAGG- ${ }^{\prime}$. All other primers have been described ${ }^{15,25,28,31}$. PCR products were identified on agarose gels by ethidium bromide staining or Southern hybridization.

\section{Received 10 June; accepted 11 August 1999.}

1. Kondo, M., Weissman, I. L. \& Akashi, K. Identification of clonogenic common lymphoid progenitor in mouse bone marrow. Cell 91, 661-672 (1997).

2. Hardy, R. R., Carmack, C. E., Shinton, S. A., Kemp, J. D. \& Kayakawa, K. Resolution and characterization of pro-B and pre-pro-B cell stages in normal mouse bone marrow. J. Exp. Med. 173, 1213-1225 (1991).

3. Rolink, A., Grawunder, U., Winkler, T. H., Karasuyama, H. \& Melchers, F. IL-2 receptor $\alpha$ chain (CD25,TAC) expression defines a crucial stage in pre-B cell development. Int. Immunol. 6, 1257-1264 (1994).

4. Rolink, A., Kudo, A., Karasuyama, H., Kikuchi, Y. \& Melchers, F. Long-term proliferating early pre B cell lines and clones with the potential to develop to surface Ig-positive, mitogen rective B cells in vitro and in vivo. EMBO J. 10, 327-336 (1991).

5. Thévenin, C., Nutt, S. L. \& Busslinger, M. Early function of Pax5 (BSAP) prior to the pre-B cell receptor stage of B lymphopoiesis. J. Exp. Med. 188, 735-744 (1998).

6. Zhuang, Y., Soriano, P. \& Weintraub, H. The helix-loop-helix gene E2A is required for B cell formation. Cell 79, 875-884 (1994).

7. Bain, G. et al. E2A proteins are required for proper B cell development and initiation of immunoglobulin gene rearrangements. Cell 79, 885-892 (1994).

8. Lin, H. \& Grosschedl, R. Failure of B-cell differentiation in mice lacking the transcription factor EBF Nature 376, 263-267 (1995).

9. Sigvardsson, M., O'Riordan, M. \& Grosschedl, R. EBF and E47 collaborate to induce expression of the endogenous immunoglobulin surrogate light chain genes. Immunity 7, 25-36 (1997).

10. Kee, B. L. \& Murre, C. Induction of early B cell factor (EBF) and multiple B lineage genes by the basic helix-loop-helix transcription factor E12. J. Exp. Med. 188, 699-713 (1998).

11. Busslinger, M. \& Nutt, S. L. in Molecular Biology of B-Cell and T-Cell Development (eds Monroe, J. G. \& Rothenberg, E. V.) 83-110 (Humana, Totowa, New Jersey, 1998).

12. Adams, B. et al. Pax-5 encodes the transcription factor BSAP and is expressed in B lymphocytes, the developing CNS, and adult testis. Genes Dev. 6, 1589-1607 (1992).

13. Li, Y.-S., Wasserman, R., Hayakawa, K. \& Hardy, R. R. Identification of the earliest B lineage stage in mouse bone marrow. Immunity 5, 527-535 (1996).

14. Urbánek, P., Wang, Z.-Q., Fetka, I., Wagner, E. F. \& Busslinger, M. Complete block of early B cell differentiation and altered patterning of the posterior midbrain in mice lacking Pax5/BSAP. Cell 79, 901-912 (1994).

15. Nutt, S. L., Urbánek, P., Rolink, A. \& Busslinger, M. Essential functions of Pax5 (BSAP) in pro-B cell development: difference between fetal and adult B lymphopoiesis and reduced $V$-to- $D J$ recombination at the IgH locus. Genes Dev. 11, 476-491 (1997).

16. Nutt, S. L., Morrison, A. M., Dörfler, P., Rolink, A. \& Busslinger, M. Identification of BSAP (Pax-5) target genes in early B-cell development by loss- and gain-of-function experiments. EMBO J. 17, 2319-2333 (1998).

17. Shinkai, Y. et al. RAG-2-deficient mice lack mature lymphocytes owing to inability to initiate $V(D)$ J rearrangement. Cell 68, 855-867 (1992).

18. Rolink, A., Melchers, F. \& Andersson, J. The SCID but not the RAG-2 gene product is required for $\mathrm{S} \mu$ S€ heavy chain class switching. Immunity 5, 319-330 (1996).

19. Yamane, T. et al. Development of osteoclasts from embryonic stem cells through a pathway that is c-fms but not c-kit dependent. Blood 90, 3516-3523 (1997).

20. Inaba, K. et al. Generation of large numbers of dendritic cells from mouse bone marrow cultures supplemented with granulocyte/macrophage colony-stimulating factor. J. Exp. Med. 176, 1693-1702 (1992).
21. Branchereau, J. \& Steinman, R. M. Dendritic cells and the control of immunity. Nature 392, 245-252 (1998).

22. Kong, Y.-Y. et al. OPGL is a key regulator of osteoclastogenesis, lymphocyte development and lymphnode organogenesis. Nature 397, 315-323 (1999).

23. Liu, F., Poursine-Laurent, J., Wu, H. Y. \& Link, D. C. Interleukin-6 and the granulocyte colonystimulating factor receptor are major independent regulators of granulopoiesis in vivo but are not required for lineage commitment or terminal differentiation. Blood 90, 2583-2590 (1997).

24. Ogasawara, K. et al. Requirement for IRF-1 in the microenvironment supporting development of natural killer cells. Nature 391, 700-703 (1998).

25. Rolink, A. et al. A subpopulation of B220 ${ }^{+}$cells in murine bone marrow does not express CD19 and contains natural killer cell progenitors. J. Exp. Med. 183, 187-194 (1996).

26. Raulet, D. H. Development and tolerance of natural killer cells. Curr. Opin. Immunol. 11, 129-134 (1999).

27. Liao, N.-S., Bix, M., Zijlstra, M., Jaenisch, R. \& Raulet, D. MHC class I deficiency: susceptibility to natural killer (NK) cells and impaired NK activity. Science 253, 199-202 (1991).

28. Rolink, A. G., Nutt, S. L., Melchers, F. \& Busslinger, M. Long-term in vivo reconstitution of T cell development by Pax5-deficient B cell progenitors. Nature 401, 603-606 (1999).

29. Wang, Z.-Q. et al. Bone and haematopoietic defects in mice lacking c-fos. Nature 360, 741-745 (1992).

30. Grigoriadis, A. et al. c-Fos: a key regulator of osteoclast-macrophage lineage determination and bone remodeling. Science 266, 443-448 (1994).

31. Hu, M. et al. Multilineage gene expression precedes commitment in the hematopoietic system. Genes Dev. 11, 774-785 (1997).

32. Borrello, M. A. \& Phipps, R. P. The B/macrophage cell: an elusive link between $\mathrm{CD}^{+}$B lymphocytes and macrophages. Immunol. Today 17, 471-475 (1996).

33. Akashi, K. et al. Simultaneous occurrence of myelomonocytic leukemia and multiple myeloma: involvement of common leukemic progenitors and their developmental abnormality of "lineage infidelity". J. Cell Physiol. 148, 446-456 (1991).

34. Klinken, S. P., Alexander, W. S. \& Adams, J. M. Hematopoietic lineage switch: v-raf oncogene converts E $\mu-m y c$ transgenic B cells into macrophages. Cell 53, 857-867 (1988).

35. Strasser, A., Elefanty, A. G., Harris, A. W. \& Cory, S. Progenitor tumours from E $\mu$-bcl-2-myc transgenic mice have lymphomyeloid differentiation potential and reveal developmental differences in cells survival. EMBO J. 15, 3823-3824 (1996).

36. Davidson, W. F., Pierce, J. H., Rudikoff, S. \& Morse III, H. C. Relationships between B cell and myeloid differentiation: studies with a B lymphocyte progenitor line, HAFTL-1. J. Exp. Med. 168, 389-407 (1988).

37. Principato, M. et al. Transformation of murine bone marrow cells with combined v-raf-v-myc oncogenes yields clonally related mature B cells and macrophages. Mol. Cell. Biol. 10, 3562-3568 (1990).

38. Borzillo, G. V., Ashmun, R. A. \& Sherr, C. J. Macrophage lineage switching of murine early pre-B lymphoid cells expressing transduced fms genes. Mol. Cell. Biol. 10, 2703-2714 (1990).

39. Cumano, A., Paige, C. J., Iscove, N. N. \& Brady, G. Bipotential precursors of B cells and macrophages in murine fetal liver. Nature 356, 612-615 (1992).

40. Kawamoto, H., Ohmura, K. \& Katsura, Y. Direct evidence for the commitment of hematopoietic stem cells to T, B and myeloid lineages in murine fetal liver. Int. Immunol. 9, 1011-1019 (1997).

41. Aiba, Y. \& Ogawa, M. Development of natural killer cells, B lymphocytes, macrophages, and mast cells from single hematopoietic progenitors in culture of murine fetal liver cells. Blood 90, 3923-3930 (1997).

42. Lacaud, G., Carlsson, L. \& Keller, G. Identification of a fetal hematopoietic precursor with B cell, T cell, and macrophage potential. Immunity $9,827-838$ (1998).

43. Galy, A., Travis, M., Cen, D. \& Chen, B. Human T, B, natural killer, and dendritic cells arise from a common bone marrow progenitor cell subset. Immunity 3, 459-473 (1995).

44. van Freeden-Jeffry, U. et al. Lymphopenia in interleukin (IL)-7 gene-deleted mice identifies IL-7 as a nonredundant cytokine. J. Exp. Med. 181, 1519-1526 (1995).

45. Enver, T. \& Greaves, M. Loops, lineages, and leukemia. Cell 94, 9-12 (1998).

46. Åkerblad, P., Rosberg, M., Leanderson, T. \& Sigvardsson, M. The B29 (immunoglobulin $\beta$-chain) gene is a genetic target for early B-cell factor. Mol. Cell. Biol. 19, 392-401 (1999).

47. Metcalf, D. Lineage commitment and maturation in hematopoietic cells: the case for extrinsic regulation. Blood 92, 345-348 (1998).

48. Enver, T., Heyworth, C. M. \& Dexter, T. M. Do stem cells play dice? Blood 92, 348-351 (1998).

49. Nutt, S. L. et al. Independent regulation of the two Pax5 alleles during B-cell development. Nature Genet. 21, 390-395 (1999).

50. Karasuyama, H. \& Melchers, F. Establishment of mouse cell lines which constitutively secrete large quantities of interleukin 2, 3, 4 and 5, using modified cDNA expression vectors. Eur. J. Immunol. 18, 97-104 (1988).

\section{Acknowledgements}

We thank K. Matsuo and E. F. Wagner for ${\mathrm{c}-f o s^{-1-}}^{-}$mice, ST2-TRANCE cells and advice concerning osteoclast biology; F. Alt for RAG2 $2^{-1-}$ mice; P. Steinlein and M. Dessing for FACS sorting and assistance with photography; and $\mathrm{H}$. Beug for critical reading of the manuscript. This work was supported by the I.M.P. Vienna, by a grant from the Austrian Industrial Research Promotion Fund and by the Basel Institute for Immunology.

Correspondence and requests for material should be addressed to M.B.

(e-mail: busslinger@nt.imp.univie.ac.at). 